\title{
A MATRIX EQUATION RELATED TO A NON-OSCILLATION CRITERION AND LIAPUNOV STABILITY
}

\author{
By WILLIAM T. REID (State University of Iowa)
}

1. Introduction. In this note it is shown that recent work of the author on Riccati matrix differential equations and non-oscillation criteria for associated linear differential systems, $[6 ; \S 7$, in particular], implies a result on the solution of an algebraic matrix equation that is intimately related to the existence of a Liapunov function for linear differential systems with constant coefficients (see, for example, Hahn [2; §8], Bellman [1; Ch. 13], or LaSalle and Lefschetz $[4 ; \S 17])$.

Matrix notation is used throughout; in particular, matrices of one column are termed vectors. The symbol $E_{n}$ is used for the $n \times n$ identity matrix, while 0 is used indiscriminately for the zero matrix of any dimensions; the conjugate transpose of a matrix $M$ is denoted by $M^{*}$. The notation $M \geq N$ or $N \leq N,\{M>N$ or $N<M\}$, is used to signify that $M$ and $N$ are Hermitian matrices of the same dimensions and $M-N$ is non-negative, \{positive\}, definite.

The basic result of this paper is as follows.

Theorem A. If $A$ and $B$ are constant $n \times n$ matrices with $B$ Hermitian and $B \geq 0$, while the $n \times n^{2}$ matrix

$$
\left\|B A B A^{2} B \cdots A^{n-1} B\right\|
$$

has rank $n$, then there exist Hermitian matrices $W_{\infty} \leq 0$ and $W_{-\infty} \geq 0$ that are extreme solutions of the matrix equation

$$
W A+A^{*} W+W B W=0
$$

in the sense that $W=W_{\infty}$ and $W=W_{-\infty}$ are individually solutions of (1.2), while if $W$ is any Hermitian matrix satisfying (1.2) then $W_{\infty} \leq W \leq W_{-\infty}$. Moreover, $W_{-\infty}>0$, $\left\{W_{\infty}<0\right\}$, if and only if all proper values $\lambda$ of $A,\{-A\}$, have $\operatorname{Re} \lambda<0$.

The proof of Theorem $\mathrm{A}$ is presented in Sect. 2, and Sect. 3 contains a modification of this result that holds when (1.1) has rank $n-k, 0<k<n$. It is worth noting that the condition that a matrix (1.1) have rank $n$ has appeared in certain treatments of optimal control problems. In particular, this condition is equivalent to the requirement that the autonomous control problem

$$
\dot{x}(t)=A x(t)+B u(t)
$$

be proper in the sense of LaSalle [5; $\$ 4$ ], or is completely controllable in the terminology of Kalman, Ho and Narendra in [3].

2. Proof of Theorem A. By Theorem 7.2 of Reid [6], a linear vector differential system

$$
u^{\prime}=A u+B v, \quad v^{\prime}=C u-A^{*} v^{\prime}
$$

with constant coefficient matrices satisfying

$$
C^{*}=C, \quad B^{*}=B \geq 0,
$$

*Received May 13, 1964. This research was supported by the Air Force Office of Scientific Research, under grant AF-AFOSR-438-63. 
and which is identically normal, is non-oscillatory on $(-\infty, \infty)$ if and only if there is an Hermitian matrix $W$ satisfying the algebraic matrix equation

$$
W A+A^{*} W+W B W-C=0 .
$$

Moreover, if such a system $(2.1)$ is non-oscillatory on $(-\infty, \infty)$ then there exist Hermitian matrices $W_{\infty}$ and $W_{-\infty}$ which are individually solutions of (2.3), and are extreme solutions for the Riccati differential equation

$$
W^{\prime}(x)+W(x) A+A^{*} W(x)+W(x) B W(x)-C=0
$$

in the sense that if $W(x)$ is any Hermitian solution of $(2.4)$ on $(-\infty, \infty)$ then $W_{\infty} \leq$ $W(x) \leq W_{-\infty}$; in particular, if $W$ is any Hermitian solution of (2.3) then $W_{\infty} \leq W \leq W_{-\infty}$. Moreover, if $\left(U_{0}(x), V_{0}(x)\right)$ is the solution of the corresponding matrix differential system

$$
U^{\prime}=A U+B V, \quad V^{\prime}=C U-A^{*} V,
$$

satisfying $U_{0}(0)=0, V_{0}(0)=E_{n}$, then $U_{0}(x)$ is non-singular for $x \neq 0$ and $W_{0}(x)=$ $V_{0}(x) U_{0}^{-1}(x)$ is such that $W_{0}(x) \rightarrow W_{\infty},\left\{W_{0}(x) \rightarrow W_{-\infty}\right\}$, as $x \rightarrow-\infty,\{x \rightarrow \infty\}$.

By definition, a system (2.1) is identically normal if the only solution $(u(x), v(x))$ of this system with $u(x) \equiv 0$ on a non-degenerate interval is the identically vanishing solution $u(x) \equiv 0, v(x) \equiv 0$. This condition is clearly equivalent to the condition that the fundamental matrix $Z(x)=\exp \left\{-x A^{*}\right\}$ of $z^{\prime}=-A^{*} z$ is such that for any constant vector $\xi$ the vector function $B Z(x) \xi$ is identically zero on a nondegenerate interval only if $\xi=0$, and in view of the Cayley-Hamilton theorem one has the following result.

LEMMA 1. A system (2.1) with constant coefficient matrices satisfying (2.2) is identically normal if and only if the matrix (1.1) is of rank $n$.

Note that if $\eta$ is a proper vector of $A^{*}$ corresponding to a proper value $\lambda$, and $B \eta=0$, then $B A^{* i} \eta=0,(j=1,2, \cdots)$, so that the following result is immediate.

Lemma 2. If $A$ and $B$ are constant $n \times n$ matrices with $B \geq 0$, the matrix (1.1) is of rank $n$, and $\eta$ is a proper vector of $A^{*}$, then $\eta^{*} B \eta>0$.

Now consider a system (2.1) for which condition (2.2) holds and also $C \geq 0$. Such a system is non-oscillatory on $(-\infty, \infty)$; that is, if $(u(x), v(x))$ is a solution with $u\left(x_{1}\right)=$ $0=u\left(x_{2}\right),\left(x_{1}<x_{2}\right)$, then $u(x) \equiv 0$ on $\left[x_{1}, x_{2}\right]$. This result, which is a special case of Theorem 5.2 of Reid [6], is a direct consequence of the observation that if $(u(x), v(x))$ is such a solution then

$$
0=\left.v^{*}(x) u(x)\right|_{x_{1}} ^{x_{*}}=\int_{x_{1}}^{x_{4}}\left[v^{*}(x) B v(x)+u^{*}(x) C u(x)\right] d x ;
$$

hence $C u(x)=0$ and $B v(x)=0$ throughout $\left[x_{1}, x_{2}\right]$, so that $u^{\prime}=A u$ and $u(x) \equiv 0$ as $u\left(x_{1}\right)=0$.

In particular, consider a system

$$
u^{\prime}=A u+B v, \quad v^{\prime}=-A^{*} v,
$$

where $A$ and $B$ are $n \times n$ matrices with $B^{*}=B \geq 0$; that is, a system (2.1) satisfying (2.2) and with $C=0$. In view of the above result and Lemma 1 , it follows that under the hypotheses of Theorem A the system $\left(2.1_{0}\right)$ is identically normal and non-oscillatory on $(-\infty, \infty)$, and thus by Theorem 7.2 of Reid [6] there exist Hermitian matrices $W_{\infty}, W_{-} \infty$ which are individually solutions of (1.2), and such that if $W$ is any Hermitian solution 
of this matrix equation then $W_{\infty} \leq W \leq W_{-\infty}$; in particular, $W=0$ is an Hermitian solution of (1.2) so that $W_{\infty} \leq 0 \leq W_{-\infty}$. Finally, for $C=0$ the solution $\left(U_{0}(x), V_{0}(x)\right)$ of (2.5) satisfying $U_{0}(0)=0, V_{0}(0)=E_{n}$ is

$$
V_{0}(x)=e^{-x A^{*}}, \quad U_{0}(x)=V_{0}^{*-1}(x) \int_{0}^{x} V_{0}^{*}(s) B V_{0}(s) d s,
$$

so that for $x \neq 0$,

$$
\begin{aligned}
W_{0}(x)=V_{0}(x) U_{0}^{-1}(x) & =V_{0}(x)\left[\int_{0}^{x} V_{0}^{*}(s) B V_{0}(s) d s\right]^{-1} V_{0}^{*}(x), \\
& =\left[\int_{0}^{x} V_{0}^{*-1}(x) V_{0}^{*}(s) B V_{0}(s) V_{0}^{-1}(x) d s\right]^{-1},
\end{aligned}
$$

and hence for $x \neq 0$,

$$
W_{0}(x)=\left[\int_{0}^{x} e^{(x-s) A} B e^{(x-s) A^{*}} d s\right]^{-1}=\left[\int_{0}^{x} e^{t A} B e^{t A^{*}} d t\right]^{-1} .
$$

In particular, $W_{0}\left(x_{1}\right)>W_{0}\left(x_{2}\right)>0$ for $0<x_{1}<x_{2}$, and $W_{-\infty}=\lim _{x \rightarrow \infty} W_{0}(x)$ is positive definite if and only if

$$
\lim _{x \rightarrow \infty} \eta^{*}\left[\int_{0}^{x} e^{t A} B e^{t A^{*}} d t\right] \eta
$$

is finite for arbitrary constant vectors $\eta$. Now for $\eta$ a proper vector of $A^{*}$ corresponding to a proper value $\lambda=\alpha+i \beta$ we have

$$
\eta^{*}\left[\int_{0}^{x} e^{t A} B e^{t A^{*}} d t\right] \eta=\left(\eta^{*} B \eta\right) \int_{0}^{x} e^{2 \alpha t} d t,
$$

and consequently if $W_{-\infty}$ is positive definite then all proper values $\lambda$ of $A^{*}$ have $\operatorname{Re} \lambda<0$; as $\lambda_{0}$ is a proper value of $A^{*}$ if and only if $\bar{\lambda}_{0}$ is a proper value of $A$, this condition is equivalent to $R e \lambda<0$ for all proper values $\lambda$ of $A$. Conversely, if $R e \lambda<0$ for all proper values $\lambda$ of $A$, then

$$
\int_{0}^{\infty} e^{t A} B e^{t A^{*}} d t=\lim _{x \rightarrow \infty} \int_{0}^{x} e^{t A} B e^{t A^{*}} d t
$$

is a positive definite matrix, and $W_{-\infty}$, the inverse of this matrix, is positive definite. The conclusion that $W_{\infty}<0$ if and only if all proper values $\lambda$ of $-A$ have $\operatorname{Re} \lambda<0$ follows by a similar argument.

Clearly $W$ is a positive definite solution of (1.2) if and only if $\Omega=W^{-1}$ is a positive definite solution of

$$
A \Omega+\Omega A^{*}=-B .
$$

For $A$ and $B$ real matrices, and $B$ positive definite, (2.7) is a well-known matrix equation occurring in the determination of a Liapunov function for a linear vector differential equation with constant coefficients, (see, for example, Hahn [2; §8], Bellman [1; Ch. 13], or LaSalle and Lefschetz $[4 ; \S 17])$.

3. An extension of Theorem A. If the matrix (1.1) is not of rank $n$ then the system (2.1) is not identically normal, and the result of Theorem A is modified. Now for nonoscillatory systems that are not identically normal the author [7] has recently discussed 
the existence of principal solutions and related distinguished solutions of associated Riccati equations, and the results of that paper might be used to obtain a generalization of Theorem A. For the case of the above systems with constant coefficients, however, this may be done directly as follows.

Theorem B. Suppose that $A$ and $B$ are constant $n \times n$ matrices with $B$ Hermitian and $B \geq 0$, while the matrix (1.1) has rank $n-k, 0<k<n$. If $\Delta$ is an $n \times k$ matrix of rank $k$ such that

$$
0=\Delta^{*} B=\Delta^{*} A^{i} B, \quad(j=1,2, \cdots, n-1),
$$

and $Q$ is an $n \times(n-k)$ matrix such that $\Delta^{*} Q=0, Q^{*} Q=E_{n-k}$, and $Q=Q^{*} A Q$, $B=Q^{*} B Q$, then there exist $(n-k) \times(n-k)$ Hermitian matrices $W_{\infty} \leq 0, W_{-\infty} \geq 0$ which are extreme solutions of the matrix equation

$$
W Q+Q * W+W B W=0
$$

in the sense that $W=W_{\infty}$ and $W=W_{-\infty}$ are individually solutions of (3.2), and if $W$ is any Hermitian matrix satisfying (3.2) then $W_{\infty} \leq W \leq W_{-\infty}$; moreover, $W_{-\infty}>0$, $\left\{W_{\infty}<0\right\}$, if and only if all proper values $\lambda$ of $\alpha,\{-Q\}$, have Re $\lambda<0$.

Without loss of generality it may be supposed that the matrix $\Delta$ satisfying (3.1) is normalized so that $\Delta^{*} \Delta=E_{k}$; in this case, $E_{n}=Q Q^{*}+\Delta \Delta^{*}$, so that $B Q^{*}=$ $Q^{*} B\left[Q Q^{*}\right] A^{*} Q=Q^{*} B\left[Q Q^{*}+\Delta \Delta^{*}\right] A^{*} Q=Q^{*} B A^{*} Q$, and by induction it follows that $B Q^{* i}=Q^{*} B A^{* i} Q,(j=1,2, \cdots)$. If $\eta$ is a vector such that $0=B \eta=B Q^{* i} \eta,(j=1,2, \cdots)$, then $\xi=Q \eta$ is such that $0=Q^{*} B \xi=Q^{*} B A^{* i} \xi$, $(j=1,2, \cdots)$, and as $\Delta^{*} B=0$ it follows that $0=B \xi=B A^{* i} \xi,(j=1,2, \cdots)$. Since $\Delta^{*} \xi=\Delta^{*} Q \eta=0$, it then follows that $\xi=0$, and hence $\eta=Q^{*} \xi=0$. That is, the set of column vectors of $B$, $B Q^{* i}$, $(j=1,2, \cdots)$, contains $n-k$ linearly independent vectors, so that by the CayleyHamilton theorem the $(n-k) \times(n-k)^{2}$ matrix

$$
\left\|B Q B Q^{2} \Theta \cdots Q^{n-k-1} \Theta\right\|
$$

has rank $n-k$, and consequently the result of Theorem $\mathrm{B}$ is an immediate corollary to Theorem A.

The basic properties that relate the conclusion of Theorem B to the general results of Reid [7] are as follows:

(i) if $\left(U_{0}(x), V_{0}(x)\right)$ is the solution of $\left(2.1_{0}\right)$ defined by (2.6), then under the hypotheses of Theorem $\mathrm{B}$ the $n \times n$ matrix

$$
K(x)=\int_{0}^{x} V_{0}^{*}\left(s-x_{0}\right) B V_{0}\left(s-x_{0}\right) d s, \quad x \neq 0,
$$

is an Hermitian matrix of rank $n-k$ and $K(x) \Delta=0$, where $\Delta$ if an $n \times k$ matrix of rank $k$ satisfying (3.1);

(ii) if $K$ is an Hermitian $n \times n$ matrix of rank $n-k, 0<k<n, \Delta$ is an $n \times k$ matrix such that $K \Delta=0, \Delta^{*} \Delta=E_{k}$, and $Q$ is an $n \times(n-k)$ matrix such that $\Delta^{*} Q=0$, $Q^{*} Q=E_{n-k}$, then the E. H. Moore general reciprocal $K^{*}$ of $K$, (see, for example, Reid $[7 ; \S 6])$, is given by $K^{*}=Q\left[Q^{*} K Q\right]^{-1} Q^{*}$.

\section{BibLIOGRAPHY}

1. R. Bellman, Introduction to matrix analysis, McGraw-Hill, 1960 
2. W. Hahn, Theorie und Anwendung der direkten Methode von Ljapunov, Ergebnisse der Mathematik und ihrer Grenzgebiete, Neue Folge, Heft 22, Springer-Verlag, 1959

3. R. E. Kalman, Y. C. Ho and K. S. Narendra, Controllability of linear dynamical systems, Contributions to Differential Equations, Vol. 1, Interscience, 1963, pp. 189-213

4. J. LaSalle and S. Lefschetz, Stability by Liapunov's direct method with applications, Academic Press, 1961

5. J. P. LaSalle, The time optimal control problem, Contributions to the Theory of non-linear Oscillations, vol. 5, Princeton University Press, 1960, pp. 1-24

6. W. T. Reid, Riccati matrix differential equations and non-oscillation criteria for associated linear differential systems, Pacific J. Math. 13 (1963) 665-685

7. W. T. Reid, Principal solutions of non-oscillatory linear differential systems, to appear in J. Math. Anal. and Appl.

\section{CONDITIONS FOR THE CAUSALITY OF NONLINEAR OPERATORS DEFINED ON A FUNCTION SPACE*}

By I. W. SANDBERG (Bell Telephone Laboratories, Inc., Murray Hill, New Jersey)

Abstract. This note considers nonlinear operators $T$ with both range and domain subsets of the space of complex $n$-vector-valued functions of the real variable $t$ for $-\infty<t<\infty$. Conditions, in which energy-type quantities play a key role, are presented under which $T$ is causal in the following sense:

Let $D(T)$ denote the domain of $T$ and let $t_{0}=\sup \left\{t^{\prime} \mid\right.$ for all $f \varepsilon D(T), f(t)=0$ for almost all $\left.t<t^{\prime}\right\}$. Then $T$ is causal if for an arbitrary $\delta>t_{0}, T f=T g$ a.e. on $\left(t_{0}, \delta\right)$ whenever $f$ and $g$ belong to $D(T)$ and $f=g$ a.e. on $\left(t_{0}, \delta\right)$.

1. Notation. Let $\mathfrak{H C}_{n}$ denote the space of complex measurable $n$-vector-valued functions of the real variable $t$ for $-\infty<t<\infty$. The complex-conjugate transpose of an arbitrary $f \varepsilon \mathcal{F}_{n}$ is written as $f^{*}$. With $g$ and $h$ arbitrary elements of $\mathfrak{H}_{n}$, and $x$ either an arbitrary real number or $\infty$, let $\langle g, h ; x\rangle$ denote

$$
\int_{-\infty}^{x} g^{*} h d t
$$

and let

$$
\|g ; x\|=(\langle g, g ; x\rangle)^{1 / 2} .
$$

The symbol $R$ denotes the set of real-valued functions and

$$
\mathfrak{L}_{2 n}=\left\{f \mid f \varepsilon \mathcal{K}_{n},\langle f, f ; \infty\rangle<\infty\right\} .
$$

If $f \varepsilon \mathfrak{F C}_{n}$, and $x<\infty$, then $f_{x}$ is defined by

$$
\begin{aligned}
f_{x}=f, & t \leq x \\
f_{x}=0, & t>x .
\end{aligned}
$$

2. Introduction. The external properties of a physical system can frequently be characterized by an operator relation of the form

$$
g=T f,
$$

*Received February 26, 1964; revised manuscript received July 2, 1964. 